\title{
Early Detection of Abnormal Patient Arrivals at Hospital Emergency Department
}

\author{
(presented at the $6^{\text {th }}$ IESM Conference, October 2015, Seville, Spain) (c) $\mathrm{I}^{4} \mathrm{e}^{2} 2015$
}

\author{
Fouzi Harrou, Ying Sun, \\ CEMSE Division, \\ King Abdullah University of Science and Technology \\ Thuwal 23955-6900, Saudi Arabia \\ Email: fouzi.harrou@kaust.edu.sa
}

\author{
Farid Kadri, Sondès Chaabane, Christian Tahon \\ LAMIH, UMR CNRS 8201, \\ University of Valenciennes and Hainaut-Cambrésis, \\ UVHC, Le Mont Houy, France, \\ Email:farid.kadri@univ-valenciennes.fr
}

\begin{abstract}
Overcrowding is one of the most crucial issues confronting emergency departments (EDs) throughout the world. Efficient management of patient flows for ED services has become an urgent issue for most hospital administrations. Handling and detection of abnormal situations is a key challenge in EDs. Thus, the early detection of abnormal patient arrivals at EDs plays an important role from the point of view of improving management of the inspected EDs. It allows the EDs mangers to prepare for high levels of care activities, to optimize the internal resources and to predict enough hospitalization capacity in downstream care services. This study reports the development of statistical method for enhancing detection of abnormal daily patient arrivals at the ED, which able to provide early alert mechanisms in the event of abnormal situations. The autoregressive moving average (ARMA)-based exponentially weighted moving average (EWMA) anomaly detection scheme proposed was successfully applied to the practical data collected from the database of the pediatric emergency department (PED) at Lille regional hospital center, France.
\end{abstract}

\section{INTRODUCTION}

Nowadays, with the growing demand for emergency medical care, the management of hospital emergency departments (EDs) has become increasingly important [1], [2], [3], [4]. The anticipation and/or management of patient influx is one of the most crucial problems in emergency departments (EDs) throughout the world. To deal with this influx of patients, EDs require significant human and material resources, as well as a high degree of coordination among human and material elements [5]. Unfortunately, these resources are limited. The consequences of this influx of patients has resulted in problems of ED overcrowding [6]. Consequently, EDs managers need to continuously monitor the ED behaviour and abnormal situations that may be occurs by using control strategies. These strategies may help managers to control the problems related to the demand and to organize resources better.

Handling abnormal situations is a key challenge in building safe and reliable EDs. Detection of abnormal situation plays an important role from the point of view of improving management, reliability, and safety of the inspected emergency department. The purpose of abnormal situation detection is to identify any event indicating a distance from the ED behaviour compared to its normal behaviour. For example, detection of significant patient flows, allows to ED to manage and optimize the internal resources, and to predict enough hospitalization capacity in downstream being able to absorb the occasional flow without pledge the quality of care for patients.

This paper presents statistical technique for detecting signs of abnormal situation generated by the influx of patients at emergency department. The remainder of this paper is organized as follows: Section II briefly describes the state of the art in time-series modelling and SPC-based monitoring techniques. Section III outlines the methodology used for the detection of abnormal situations in EDs. Section IV presents the application of the proposed methodology in the early detection of abnormal patient arrivals at the PED in Lille regional hospital centre, France. Finally, Section V reviews the main points discussed in this work and concludes the study.

\section{STATE OF THE ART}

This section presents briefly a time series analysis and how it can be used for predicting in a hospital emergency departments, and the monitoring techniques based on the statistical process control (SPC).

\section{A. Time Series Analysis}

The literature review shows that time series analysis has been largely applied in the hospital field to forecast the patient arrivals (day, month, year), length of stay, and for projecting the utilization of inpatient days. In this regard, some of the research efforts made by the some researchers deserve mentioning. [7] used three statistical methods (moving average and seasonal decomposition methods) to predict the number of ED presentations at any hour of the week in a university hospital in New Mexico. [8] developed a statistical model for an emergency department at a hospital in Israel based on 3 years of daily time series data. They used a regression model with a linear trend, and 3 types of seasonal factors that represent the effects of the day of the week, the month of the year, and the type of day (holiday, half working day, or full working day). [9] employed two univariate time-series analysis to model and forecast the monthly patient volume over the interval 1986 to 1996 at the family and community medicine primary health care clinic of King Faisal University, Al-Khobar, Saudi Arabia. [10] used BoxJenkins models for forecasting the number of daily emergency admissions, and 
the number of beds occupied by emergency admissions on a daily basis at Bromley Hospitals NHS Trust in United Kingdom. [11] studied the patterns of ED at a hospital in Tenerife, Spain. Their analysis is based on a time series of the number of ED presentations each hour over a 6-year (1997 to 2002). The authors divided time series models to linear models and nonlinear ones. They discussed about strength and weakness of each method. [12] used two statistical methods: i) an exponential smoothing and ii) BoxJenkins Method to forecast the number of patients who present each month from 2000 to 2005 at the ED of a hospital in regional Victoria, Australia. [13] evaluated the use of four statistical methods (BoxJenkins, time series regression, exponential smoothing, and artificial neural network models) to predict daily ED patient volumes of 27 months (from 2005 to 2007) at three diverse hospital EDs that operate in Utah and southern Idaho (USA). The authors compared the use of the four models to a benchmark multiple linear regression model already available in the emergency medicine literature. [14] used time series method to predict daily emergency department attendances, the authors have applied separately the autoregressive integrated moving average (ARIMA) method to three acuity categories and total patient attendances at the ED of an acute care regional general hospital from July 2005 to Mar 2008. The authors have concluded that time series analysis provide a useful, readily available tool for predicting emergency department workload that can be used to plan staff roster and resource planning.

The results presented in the literature show that ARMA model [15], [16] and its variants [17] provides an analytical tool of time series very accessible both in terms of methodological constraints and the level of mathematical models used, which are less complex linear stochastic equations. In this study, ARMA model was used to describe the variation in demand for emergency care in an ED system.

\section{B. Statistical Process Control Schemes}

Statistical process control (SPC) has been widely used in various industrial processes for monitoring process and improving product quality. Statistical controls charts are one of the most commonly used tools in SPC, and have been extensively used in the quality engineering as monitoring tool to detect the presence of possible anomalies in the mean or variance of process measurements. Control charts play a crucial role in detecting whether a process is still working under normal operation conditions (usually termed in-control) or not (out-control). In other words, a control chart is a picture of a process over time that helps to identify the state of the monitored process, i.e., either it is running satisfactorily or not [18]. In such framework, numerous control charts have been developed to monitor a mean of process variable over time, and include the Shewhart chart [19], the cumulative summation or CUSUM chart [20], and the EWMA [21], [22], [23]. Over many decades, the primary utilization of control charts was focused on industrial quality control applications. Nowadays, the application of control charts has been extended to many areas include economics, medicine, informatics, and environmental. The Shewhart control charts were the rst proposed method of testing the hypothesis, which are very popular in statistical process control, can be effectively used to detect large shifts in the process mean [24]. A key disadvantage of
Shewhart charts, however, is that they only use the last data sample about inspected process and does not carry a memory of the previous data (i.e., ignore any potential information contained in past samples). Consequently, these charts are relatively insensitive to small or moderate changes in the process variables mean. Of course, this potentially makes Shewhart control charts less useful in monitoring process when small or moderate process mean shift abnormalities are of interest. Note that the ability to detect smaller parameter shifts can be improved by using a chart based on a statistic that corporate information from past samples in addition to current samples. These shortcomings motivate the use of other alternatives, such as the EWMA and the cumulative sum (CUSUM) charts, which are better suited to detect smaller shifts in process mean [18]. However, since the EWMA uses a weighted average of all past and current observations, it is a lot less sensitive to violating the normality assumption than CUSUM charts [25]. Also, CUSUM is relatively slow to respond to large shifts. Therefore, EWMA-based charts are an appropriate monitoring scheme to be adopted when dealing with individual observations [26], [22]. According to the literature, EWMA is one of the most frequently used control charts for monitoring autocorrelated processes because of its flexibility (allows suitable parameters to be selected to achieve the highest possible performance) and sensitivity to small shifts.

\section{TIME-SERIES Modelling-BASEd EWMA ANOMALY-Detection Method}

The early detection of atypical changes in ED behaviour can be achieved by integrating a time-series as modelling framework and analysis model EWMA detection scheme.

\section{A. Time Series Modelling}

In practice, most phenomena present a time dimension that is usually dissimulated but should be taken into account. This is the case of patient flow data for which the patient flow $Y_{t}$ at time $\mathrm{t}$ depends on previous values $\left(Y_{t-1}, Y_{t-2}, \ldots\right)$. The three univariate time-series models widely applied to model patient volume in EDs are autoregressive processes (AR), moving average processes (MA), and autoregressive moving average processes (ARMA). Adding non-stationary models to the mix leads to the integrated ARMA or autoregressive integrated moving average (ARIMA) model popularized in the work by [27]. ARMA models are very comprehensive linear models and can represent many types of linear relationships, such as autoregressive (AR), moving average (MA), and mixed AR and MA time-series structures.

The AR model is intuitively appealing because it describes how an observation directly depends upon one or more previous measurements plus white noise. For time-series observations $Y_{t}$, the AR model of order $p$, which is also written as $\operatorname{AR}(p)$, is defined by:

$$
Y_{t}=\sum_{i=1}^{p} a_{i} Y_{t-1}+\varepsilon_{t}
$$

where $a_{i}$ are non-seasonal AR parameters, and $\varepsilon_{t}$ is zeromean Gaussian noise $\varepsilon_{t} \sim \mathcal{N}\left(0, \sigma^{2}\right)$. 
MA models describe how an observation depends upon the current white noise term as well as one or more previous errors. An MA model of order $q$, also denoted by $\mathrm{MA}(q)$, is defined as:

$$
Y_{t}=\varepsilon_{t}+\sum_{j=1}^{q} b_{j} \varepsilon_{t-j}
$$

where $b_{j}$ are non-seasonal MA parameters. The ARMA model was first presented by Box and Jenkins [27]. ARMA models are more sophisticated stochastic models that combine elements of moving average methods and regression methods. One advantage of an ARMA process is that it involves fewer parameters than an MA or AR process alone [25]. The mixed AR and MA or ARMA model of order $(p, q)$, also denoted autoregressive moving average, $\operatorname{ARMA}(p, q)$ is written as:

$$
Y_{t}=\sum_{i=1}^{p} a_{i} Y_{t-1}+\sum_{j=1}^{q} b_{j} \varepsilon_{t-j}+\varepsilon_{t}
$$

where $Y_{t}$ is the variable to be predicted using previous samples of the time series, $\varepsilon_{t}$ is a sequence of i.i.d. (independent and identically distributed) terms which have zero mean. $a_{i}$ (auto-regressive part) establish a linear relationship between the value predicted by the model at time $t$ and the past values of the time series. $b_{j}$ (moving average part) establish a linear relationship between the value predicted by the model at time $t$ and a Gaussian distribution of i.i.d. samples [26].

\section{B. EWMA Control Scheme Based Monitoring}

The EWMA control scheme was first introduced by [28], and has been extensively used in time-series analysis. In EWMA control schemes, the moving average is calculated by multiplying the historical observations by a weight that decays exponentially over time [18]. The EWMA decision statistic is described by the following recursive formula:

$$
z_{t}=\lambda x_{t}+(1-\lambda) z_{t-1}
$$

where $\lambda$ is a weighted parameter, with $0<\lambda \leq 1$, and $x_{t}$ is the value of the supervised variable at time $t$. The initial value $x_{0}$ is set equal to the process in-control mean, $\mu_{0}$. Generally, small values of $\lambda$ increase the chart's sensitivity to small shifts in the process mean, while large values of $\lambda$ increase its sensitivity to large shifts [18], [29]. The standard deviation of $z_{t}$ is defined as $\sigma_{z_{t}}=\sigma_{0} \sqrt{\frac{\lambda}{(2-\lambda)}\left[1-(1-\lambda)^{2 t}\right]}$, where $\sigma_{0}$ is the standard deviation of the fault free or preliminary data set. The EWMA control scheme declares a fault when the value of $z_{t}$ does not lie within the interval between an upper, $U C L$, and lower, $L C L$, control limits. The upper and lower control limits are usually set as [18]: $U C L / L C L=\mu_{0} \pm L \sigma_{z_{t}}$, where $L$ is a multiplier of EWMA standard deviation $\sigma_{z_{t}}$. It is required that the two parameters $L$ and $\lambda$ should be carefully set [18]. A slight modification of the two-sided EWMA scheme (i.e. has upper and lower control limits) can lead to its one-sided version, which only has an upper control limit.

The early detection of changes in ED behavior can be accomplished using statistical analysis and control chart methods. To this end, a time-series analysis model was integrated with EWMA charts to develop a methodology for the monitoring and early detection of abnormal ED demands.

\section{ARMA-Based EWMA Anomaly Detection Strategy}

In this subsection, ARMA model is integrated with EWMA to develop a scheme for monitoring the occurrence of abnormal situations caused by abnormal patient arrivals at the PED.

The difference between the observed value of the daily patient attendances at the PED, $y$, and the predicted value, $\hat{y}$, obtained from ARMA model represent the residual vector, $\varepsilon_{t}=\left[\varepsilon_{1}, \varepsilon_{2}, \ldots, \varepsilon_{n}\right]$ is used as an indicator to detect a possible abnormal situations or changes in ED behaviour. The residuals are close to zero when the behavior of the monitored PED is in normal situation. However, when an abnormal situation occurs, the residuals deviate significantly from zero indicating the presence of a new situation that is significantly distinguishable from the normal situation. Then, the EWMA decision function based on the residuals obtained from ARMA model can be computed as follows:

$$
z_{t}=\lambda \varepsilon_{t}+(1-\lambda) z_{t-1}, \quad t \in[1, n]
$$

The steps of the ARMA-based EWMA detection algorithm are summarized in the Table I.

TABLE I. STEPS OF THE PROPOSED ABNORMAL SITUATION DETECTION STRATEGY.

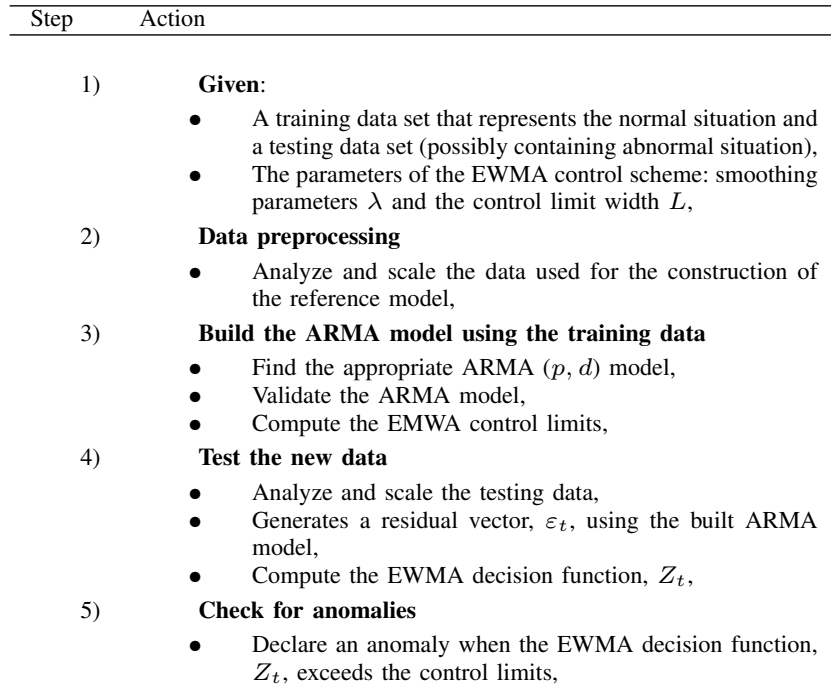

The developed ARMA-based EWMA anomaly detection strategy will be used in next section to early detection of abnormal patient arrivals at the peadiatric emergency department (PED) in Lille regional hospital centre (France).

\section{Case Study: Pediatric Emergency Department}

This retrospective study was conducted utilizing a dataset extracted from the database of the peadiatric emergency department (PED) in Lille regional hospital centre (France). The data used in this paper are the time series of daily patient attendances at PED Lille regional hospital centre in France, from January 2011 to December 2011. 


\section{A. Data Pre-treatment}

The first step in any time series analysis is to plot the observations against time to obtain simple descriptive measures of the main properties of the series. Time series plots give a preliminary understating of time behaviour of the series. The graph should show up important features of the series such as trend and seasonality. Plots of these times series and the corresponding auto-correlation functions (ACF), are shown in Figures 1 and 2, respectively.

From the time series plot depicted in Figure 1, it is apparent the data does not have a long term trend or seasonality. According to the Figure 2, one can remark that no periodicity is apparent in the ACF. Also, the ACF decreases exponentially to 0 as the lag increases. We can observe the presence of a significant short-term dependence (short-memory) in all-time series. The short-memory, or short-term dependence, describes the low-order correlation structure of a series. It is well known that the ARMA model, also termed short memory, processes, models short-term correlations in a time series.

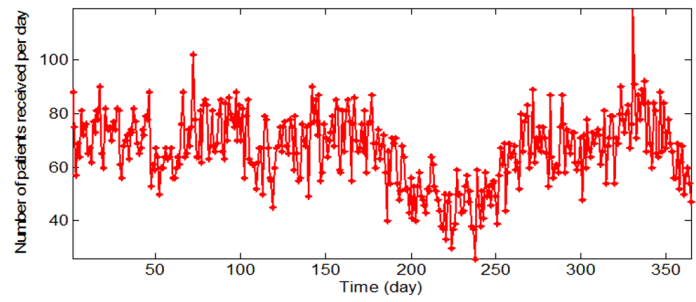

Fig. 1. Daily attendances at the PED from Jan-Dec 2011.

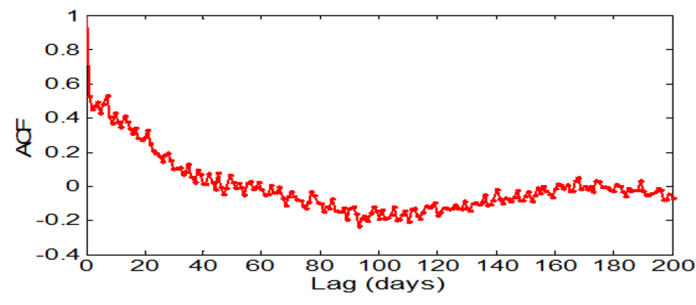

Fig. 2. ACF of daily patient attendances at the PED from Jan-Dec 2011.

\section{B. Build the ARMA Model}

To find the best ARMA model, which fits the data, Box and Jenkins's methodology [27] is used to estimate all the parameters characterizing a given time series. Model orders are fixed by analyzing the simple autocorrelation and partial autocorrelation functions of time series. The best-fit model for total attendances was $\operatorname{ARMA}(2,1)$, which is a non-seasonal and stationary auto-regression moving average model. Parameter estimation of ARMA (p, q) when orders $p$ and $q$ are assumed to be known can be achieved by various methods in the time domain. In this study, the parameters of the ARMA models have been estimated by using the method of maximum likelihood estimates for each data set [27]. The advantage of the method is that the whole data are used, thus achieving a better estimation than methods using only a subset of the data. The mathematical equation of ARMA model for the daily patient arrivals at PED is as follows:

$$
\left(1-0.22 B-0.01 B^{2}\right) Y_{t}=\mu+(1-0.23 B) \varepsilon_{t},
$$

where $B$ is the backshift operator defined as $B^{k} Y_{t}=Y_{t-k}$. To illustrate the quality of the selected ARMA-based model, one common and simple approach is to regress predicted versus observed values (or vice versa). Ideally, on a plot of observed versus predicted. The points should be scattered around a diagonal straight line $(Y=\hat{Y})$. Figure 3 shows the scatter plot of observed versus predicted values of the testing data set obtained from the selected ARMA model. As can be viewed from the Figure 3, the points follow the diagonal line (predicted $=$ observed) quite, and there is no indication of a curvature or other anomalies.

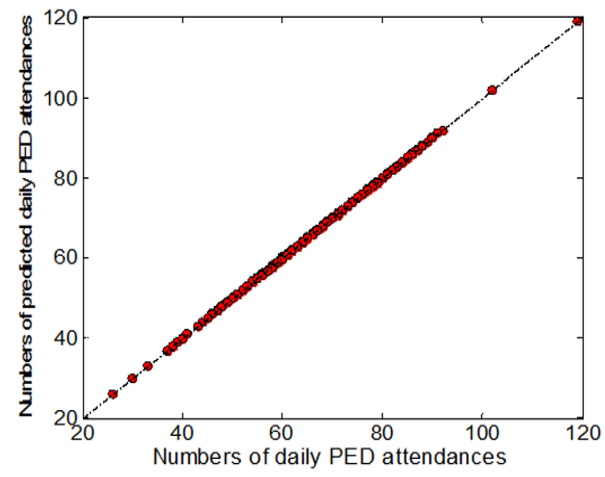

Fig. 3. Scatter plot of numbers of daily attendances at PED, observed versus predicted, Jan-Dec 2011.

1) Model Selection: To evaluate the predictive abilities of the ARMA model, several measures of a model's ability to fit data have been developed [16]. In this study, RMSE and $R^{2}$ have been used to assess the goodness of the constructed ARMA model.

For the selected model, the value of $R^{2}=0.99$ which means that $99 \%$ of the total sum of squares in the training set is explained by the model, and that only $1 \%$ is in the residuals, and the value of RMSE $=0.06$. The high $R^{2}$ and the low RMSE of the best-fit model, means that the selected model closely represented the observed time series.

2) Residuals Analysis: The residual normality hypothesis was verified in this study by examining Henrys line and the histogram. The latter is the simplest graphical tool that allows us to visually check the normality of the residuals. The verification of the normality of the residuals using Henry's line and the histogram for data time series is illustrated in Figure 4. It shows that the assumption of a normal distribution for the residuals appears to be reasonable.

The independence of the residuals (more specifically the absence of autocorrelation) was then verified. The residuals were assumed to be non-autocorrelated. To determine whether residuals are non-autocorrelated, the ACF of the residuals is examined. The ACF of the residuals for the selected model presented in Figure 5 indicates that the residuals were not significantly different from a white noise series. According to Figure 5, the residuals are approximately uncorrelated. As the residuals are normally distributed and uncorrelated, it can be deduce that the model fits the data well. 

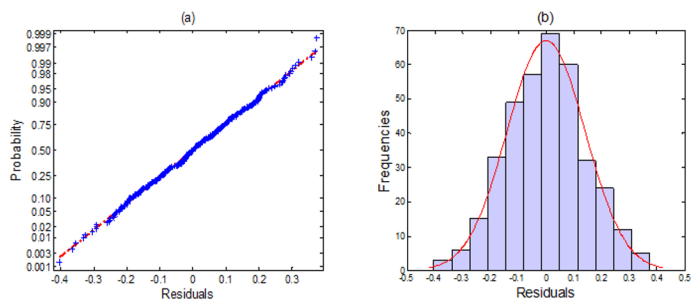

Fig. 4. Gaussian distribution test a) Henrys line Total, b) histogram.

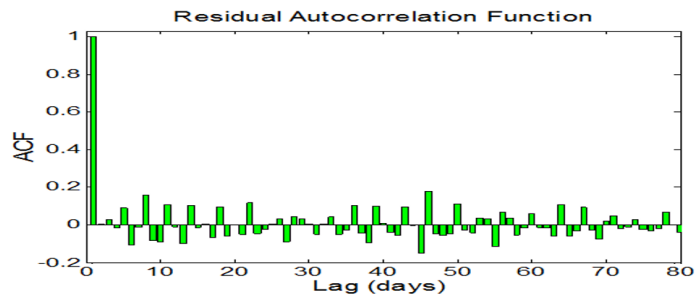

Fig. 5. ACF of residual errors.

\section{Detection Results}

In this sub-section, the ARMA model was integrated with the EWMA to develop a scheme for monitoring the occurrence of abnormal situations generated by abnormal patient arrivals at the PED. The anomaly detection abilities of the ARMAbased EWMA strategy were assessed. In the absence of testing data from the PED database used containing real anomalies, two different anomalies were simulated in this study to assess the performance of the proposed anomaly detection strategy. Additive anomalies were injected into the raw data. Two different cases of anomalies were simulated: abrupt anomaly (case A) and gradual anomaly (case B):

- $\quad$ Case A, an abrupt increase in patient arrivals at the PED was considered as a result of a sudden increase in the number of patient arrivals.

- Case B, assumed that the number of patient arrivals gradually increased and the abnormal situation slowly developed; this abnormal situation was termed gradual abnormal situation.

1) Case A: Abrupt Abnormal Situation: In this case, we investigated the problem of detecting abrupt abnormal situation. The testing dataset used in this case should first be scaled with the mean and standard deviation of the training fault free data. The developed ARMA model using the training anomalyfree data is used to detect possible anomalies using unseen testing data. Herein, the performance of ARMA-based EWMA anomaly detection method is illustrated. Three examples $\left(\mathrm{A}_{1}\right.$, $A_{2}, A_{3}$ ) are presented here to illustrate the ability of the proposed ARMA-based EWMA control scheme to detect abrupt abnormal situation.

In the first example $\left(\mathrm{A}_{1}\right)$, an additive anomaly is introduced in the $\mathrm{y}$ from samples number 200 to 300. This anomaly is represented by a constant bias of amplitude equal $30 \%$ of the total variation in $y$. The green shaded area represents the zone where the simulated abnormal situation is produced. The results of the ARMA-based EWMA anomaly detection scheme, with $\lambda=0.25$ based on testing data, which is plotted in Figure 6 clearly shows the violation of the confidence limits and thus the ability of the proposed ARMA-based EWMA anomaly detection scheme to detect correctly this abnormal situation without any false alarms.

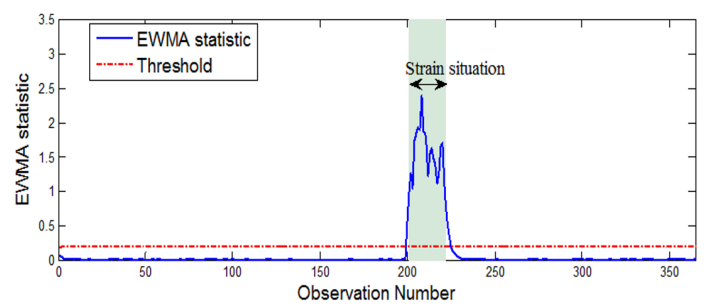

Fig. 6. The time evolution of the EWMA statistic in the presence of an abnormal situation $\left(\mathrm{A}_{1}\right)$.

In the second example $\left(\mathrm{A}_{2}\right)$, a moderate bias level, which is $30 \%$ of the total variation in testing data, is injected between samples 200 and 300. As can be seen from Figure 7, the proposed ARMA-based EWMA anomaly detection scheme could satisfactorily detect the given abnormal situation without any false alarms.

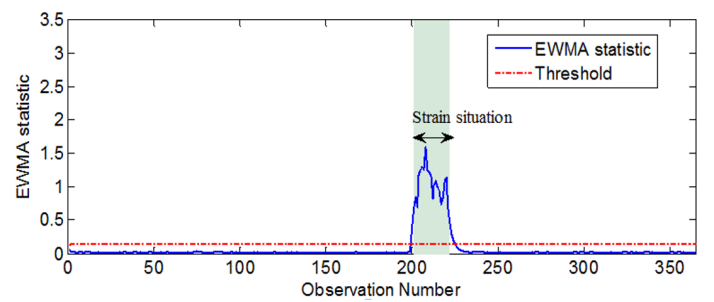

Fig. 7. The time evolution of the EWMA statistic in the presence of an abnormal situation $\left(\mathrm{A}_{2}\right)$.

In the third example $\left(\mathrm{A}_{3}\right)$, we introduce into some testing data, different bias of amplitude on the testing data $20 \%$ of the total variation in testing data from sample 150 to 170 , a bias of $30 \%$ from sample 200 to 220 , and a bias of $50 \%$ is introduced between samples 250 and 270. The results of the ARMA-based EWMA anomaly detection scheme, with $\lambda=0.2$, based on testing data, which is plotted in Figure 8 clearly shows the violation of the confidence limits and thus the ability of the proposed anomaly detection algorithm to detect correctly this stain situations without any false alarms.

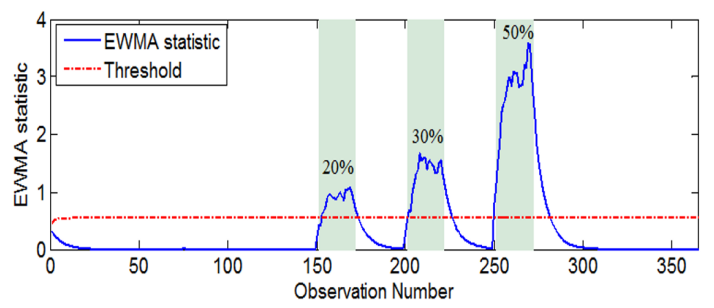

Fig. 8. The time evolution of the EWMA statistic in the presence of an abnormal situation $\left(\mathrm{A}_{3}\right)$.

2) Case B: Gradual Abnormal Situation: A slowly increasing (or ramping) abnormal situation usually indicates a slow increase in the number of patient arrivals at the PED over a long period of time. The aim of this case study was to assess 
the potential of the proposed ARMA-based EWMA control scheme in detecting slow or gradual abnormal patient arrivals. Towards this end, a slow increase in ED arrivals with a slope of 0.1 was added to the test data starting at sample number 85 of the simulated testing data. In other words, the input variable $y_{t}$ was linearly increased from sample 85 to sample number 95 of the testing data by adding $0.1(k-200)$ to the $y_{t}$ value of each sample in this range, where $k$ is the sample number. The results of the EWMA statistic are shown in Figure 9. This figure shows that ARMA-based EWMA statistic was able to successfully detect this abnormal situation by exceeding the threshold value. The ARMA-based EWMA statistic gradually increased as the demand slowly increased, and began to violate the confidence limits when the abnormal situation became sufficiently large to be detected by the given model.

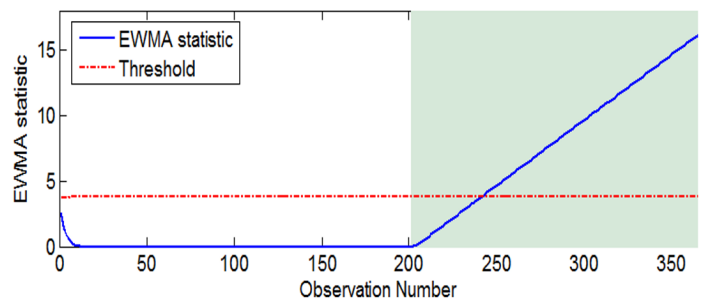

Fig. 9. The time evolution of the EWMA statistic in the presence of a gradual abnormal situation.

\section{CONCLUSiON}

In this paper, an ARMA-based EWMA anomaly detection method was presented for the early detection of abnormal patient arrivals at the paediatric emergency department (PED) in Lille regional hospital centre (France). Based on the data analysed from January to December 2011, the reference model with the best fit was a stationary auto-regression moving average model (ARMA). The results indicate that the proposed model provides an acceptable description of daily patient arrivals at the PED.

ARMA was used as the modelling framework of the ARMAbased EWMA anomaly detection methodology developed. Then, the EWMA control scheme was applied to the residuals to detect anomalies when the data did not fit the reference ARMA model. To assess the anomaly detection abilities of the proposed ARMA-based EWMA anomaly detection method, two case studies were considered, one involving an abrupt abnormal situation caused by a sudden increase in the number of patient arrivals, the second one involving a gradual abnormal situation caused by a gradual increase in patient arrivals at the PED. The simulation results of all the cases clearly show the effectiveness of the proposed ARMA-based EWMA anomaly detection methodology.

Early detection of abnormal situations in EDs is beneficial to foster reactive control thus enabling these situations to be avoided and/or the consequences reduced. The latter is important not only for anticipating and managing patients and internal resources in EDs, but also to predict sufficient hospitalization capacity downstream so as to be able to absorb the occasional increase in patient flow without reducing the quality of patient care. Therefore, by exploiting the information provided by this anomaly detection scheme, the performance of the ED can be optimized.

\section{REFERENCES}

[1] A. Kellermann, "Crisis in the emergency department," New England Journal of Medicine, vol. 355, no. 13, pp. 1300-1303, 2006.

[2] F. Harrou, F. Kadri, S. Chaabane, C. Tahon, and Y. Sun, "Improved principal component analysis for anomaly detection: Application to an emergency department," Computers \& Industrial Engineering, vol. 88, pp. 63-77, 2015.

[3] F. Kadri, C. Pach, S. Chaabane, T. Berger, D. Trentesaux, C. Tahon, and Y. Sallez, "Modelling and management of strain situations in hospital systems using an orca approach," in Proceedings of 2013 International Conference on Industrial Engineering and Systems Management (IESM). IEEE, 2013, pp. 1-9.

[4] F. Kadri, F. Harrou, S. Chaabane, and C. Tahon, "Time series modelling and forecasting of emergency department overcrowding," Journal of medical systems, vol. 38, no. 9, pp. 1-20, 2014.

[5] F. Kadri, S. Chaabane, and C. Tahon, "A simulation-based decision support system to prevent and predict strain situations in emergency department systems," Simulation Modelling Practice and Theory, vol. 42, pp. 32-52, 2014.

[6] A. Boyle, K. Beniuk, I. Higginson, and P. Atkinson, "Emergency department crowding: time for interventions and policy evaluations," Emergency medicine international, vol. 2012, 2012.

[7] D. Tandberg and C. Qualls, "Time series forecasts of emergency department patient volume, length of stay, and acuity," Annals of emergency medicine, vol. 23, no. 2, pp. 299-306, 1994.

[8] Z. Rotstein, R. Wilf-Miron, B. Lavi, A. Shahar, U. Gabbay, and S. Noy, "The dynamics of patient visits to a public hospital ed: a statistical model," The American journal of emergency medicine, vol. 15, no. 6, pp. 596-599, 1997.

[9] R. Abdel-Aal and A. Mangoud, "Modeling and forecasting monthly patient volume at a primary health care clinic using univariate timeseries analysis," Computer Methods and Programs in Biomedicine, vol. 56, no. 3, pp. 235-247, 1998.

[10] S. Jones, M. Joy, and J. Pearson, "Forecasting demand of emergency care," Health care management science, vol. 5, no. 4, pp. 297-305, 2002.

[11] G. M. Rodríguez and J. C. Hernández, "A method for ascertaining the seasonal pattern of hospital emergency department visits," Revista Española de Salud Pública, vol. 79, no. 1, pp. 5-15, 2005.

[12] R. Champion, L. Kinsman, G. Lee, K. Masman, E. May, T. Mills, M. Taylor, P. Thomas, and R. Williams, "Forecasting emergency department presentations," Australian Health Review, vol. 31, no. 1, pp. 83-90, 2007.

[13] S. Jones, A. Thomas, R. Evans, S. Welch, P. Haug, and G. Snow, "Forecasting daily patient volumes in the emergency department," Academic Emergency Medicine, vol. 15, no. 2, pp. 159-170, 2008.

[14] Y. Sun, B. Heng, Y. Seow, and E. Seow, "Forecasting daily attendances at an emergency department to aid resource planning," BMC emergency medicine, vol. 9, no. 1, p. 1, 2009.

[15] T. Bollerslev, "Generalized autoregressive conditional heteroskedasticity," Journal of econometrics, vol. 31, no. 3, pp. 307-327, 1986.

[16] S. Makridakis, S. Wheelwright, and R. Hyndman, Forecasting methods and applications. John Wiley \& Sons, 2008.

[17] R. Shumway and D. Stoffer, Time series analysis and its applications: with $R$ examples. Springer Science \& Business Media, 2010.

[18] D. C. Montgomery, "Introduction to statistical quality control," John Wiley\& Sons, New York, 2005.

[19] W. Shewhart, Economic control of quality of manufactured product. ASQ Quality Press, 1931.

[20] E. S. Page, "Continuous inspection schemes," Biometrika, pp. 100-115, 1954.

[21] J. S. Hunter, "The exponentially weighted moving average," Journal of Quality Technology, vol. 18, no. 4, pp. 203-210, 1986.

[22] F. Harrou and M. Nounou, "Monitoring linear antenna arrays using an exponentially weighted moving average-based fault detection scheme," Systems Science \& Control Engineering: An Open Access Journal, vol. 2, no. 1, pp. 433-443, 2014. 
[23] F. Harrou, M. Nounou, and H. Nounou, "A statistical fault detection strategy using pca based ewma control schemes," in 9th Asian Control Conference (ASCC). IEEE, 2013, pp. 1-4.

[24] A. Cinar and C. Undey, "Statistical process and controller performance monitoring. A tutorial on current methods and future directions," in Proceedings of the 1999 American Control Conference, vol. 4. IEEE, 1999, pp. 2625-2639.

[25] N. Draper, H. Smith, and E. Pownell, Applied regression analysis. Wiley New York, 1966, vol. 3.

[26] E. Balaguer, A. Palomares, E. Soria, and J. Martín-Guerrero, "Predicting service request in support centers based on nonlinear dynamics, arma modeling and neural networks," Expert Systems with Applications, vol. 34, no. 1, pp. 665-672, 2008.

[27] G. Box and G. Jenkins, Time series analysis: forecasting and control. John Wiley \& Sons, 2011, vol. 734.

[28] S. Roberts, "Control chart tests based on geometric moving averages," Technometrics, vol. 1, no. 3, pp. 239-250, 1959.

[29] F. Harrou, M. N. Nounou, H. N. Nounou, and M. Madakyaru, "Plsbased ewma fault detection strategy for process monitoring," Journal of Loss Prevention in the Process Industries, vol. 36, no. 1, pp. 108-119, 2015 . 